Advances in Intelligent Systems and Computing 777

Francisco Rebelo Marcelo M. Soares Editors

Advances in

Ergonomics in Design

Proceedings of the AHFE 2018 International Conference on

Ergonomics in Design, July 21-25,

2018, Loews Sapphire Falls Resort at

Universal Studios, Orlando,

Florida, USA

黛 Springer 


\title{
The Hard Life: A Pedagogical Experience in Industrial Design
}

\author{
Afonso Borges ${ }^{1(\bowtie)}$ and José Vicente $e^{1,2,3}$ \\ ${ }^{1}$ Faculdade de Artes e Letras, DCA, Universidade da Beira Interior, \\ R. Marquês de Ávila e Bolama, 6201-001 Covilhã, Portugal \\ \{afonsoborg, jmanvicente\}@gmail.com \\ ${ }^{2}$ UNIDCOM/IADE - Unidade de Investigação em Design e Comunicação, \\ Av. D. Carlos I, 4, 1200-649 Lisboa, Portugal \\ ${ }^{3}$ Faculdade de Arquitetura, CIAUD, Universidade de Lisboa, \\ R. Sá Nogueira, 1349-055 Lisboa, Portugal
}

\begin{abstract}
This paper seeks to analyze and discuss a redesign project developed by students of master's degree in industrial design. Based on the book The Hard Life, from English designer Jasper Morrison, the briefing proposed the development of concepts for new products inspired by the selection of objects in the book, and respective written interpretations. It seeks to explore the dialogue between the original objects - their use, Morrison's interpretation - and the reinterpretation made by the students considering the current material culture.

Accompanied and coordinated with Bisarro, contemporary studio dedicated to the artisan production of small series in Bisalhães black clay, UNESCO Immaterial Cultural Heritage, the project seeks to explore the boundaries and common ground between memory and contemporary times.

More than the results, it is intended to discuss proposals and approaches, from functional interpretations, formal inspiration, to the appropriation of affordance and gesture.
\end{abstract}

Keywords: Design education $\cdot$ Redesign $\cdot$ Memory $\cdot$ Industrial design Evolutive design

\section{Introduction}

This paper aims to analyze the results of an industrial design project developed by a group of graduate designers, students of the master in industrial design at the University of Beira Interior in Portugal, during the $1^{\text {st }}$ semester of 2017-18. The name comes from the book that serves as the basis for the exercise, "The Hard Life," by the English designer Jasper Morrison and published by Lars Müller, an edition that records and comments on a selection of artifacts from the collection of the National Museum of Ethnology in Lisbon.

The museum houses a collection of 42,000 objects from the five continents, presenting itself as a central element in the development of anthropological knowledge in Portugal. It has several permanent exhibitions that address the Portuguese culture. The Museum also has rich visitable archives - the Galleries of Amazonia and the Galleries 
of Rural Life - the latter with artifacts of Portuguese rural life that depict a way of life that has undergone great transformations and has practically disappeared.

The objects selected in the book refer to the Portuguese past, and include products used in rural work and homeware, to meet daily needs, most of which are functional products originated in the most diverse regions of the country, from north to south. The book idea was born from a visit by Jasper Morrison to the museum in 2012, year in which he begins a series of trips to Portugal to photograph the artifacts, seeking to register and show how beauty can come from hardship and necessity [1].

The briefing presented to the students was that, from any chosen artifact of the book, a reinterpretation should be made and a new product, suited to the present, should be created. Considering the strategy of this design degree, which has a realistic and close connection with the national industry, the work was developed in partnership with the company Bisarro, to monitor the development process and to produce small series of one or more products designed. The company, recent in the Portuguese panorama, but already with several design prizes won, develops contemporary products in black clay of Bisalhães, resorting to artisanal processes, being its main market the hotel industry.

The black clay of Bisalhães is a traditional technique of pottery that is singularized by the processes and ancestral techniques used in creation and cooking of the clay, providing it a black tonality [2]. This pottery is characteristic of the village of Bisalhães, in the municipality of Vila Real, in the north of Portugal, and was classified as Intangible Heritage of Humanity by UNESCO in 2016 and inscribed on the List of Intangible Cultural Heritage in Need of Urgent Safeguarding. Bisalhães' crockery features pieces of two types: "churra crockery" - pieces of a utilitarian character, which are coarser, less decorated, and less produced today; and "fine crockery" - pieces of a decorative character, such as jugs and vases. It is a local archaic process, traditionally involving the whole family - man, woman and children -, with the man having the tasks of working in the wheel and taking care of the cooking process in the ground oven. The women and children have the heaviest and less considered tasks, such as preparation of the clay, decorate the pieces, supply the water and the gorse necessary to cook the objects [2].

Besides the imposition of material - use of black clay -, to provide true freedom and openness in the choice of the path, no additional constraints were suggested. Students could add other materials and choose the scale of the new object, being given the freedom to change the original artifacts, which turned out to be significant in cases where the proposals seeked to fit new functionalities.

There are some assumptions in the challenge, that even if not declared, are unavoidable. The original objects refer necessarily to the collective memory and, eventually, connote the Portuguese national reality until the middle of the twentieth century; and individual memory, given that many of this type of artifacts were still present in the student's grandparents houses. It is also impossible to circumvent the point of view of the author of the book, Jasper Morrison, from what is reflected from his perspective in the choices made for the book. The objects he chooses and the interpretations made, between knowledge gathered and his innocent opinion when he sees each object for the first time, reflect his point of view. His approach is particularly important, in this case, in what he calls familiarity, where the fundamental idea is to promote the 
identification. The recognition is rendered for each one, but refers to the knowledge accumulated over generations and rooted in the culture by repeated coexistence. Hara [3] talks about dealing with the shared values, stable and mature products and Enzo Mari in designer's role as guardian of the collective knowledge [4]. Morrison believes this link to what we know, which is familiar, gives us comfort. In this case, the familiarity that exists between the artifacts and the Portuguese people, Morrison adds his external look of someone who, being English, didn't grow up surrounded by these artefacts, which extends and frees up his vision, and is reflected in the comments made about what each, in his opinion, affords.

In addition to Jasper Morrison's choices, which serve as the first filter between the museum and the students, there is still a second choice, that of the students, reflecting their own point of view. This not only translates the point of view of each individual, but also the sum of the 28 choices reflects the view of the class as a group of design students in a particular course of industrial design. Sudjic [5] recalls how no collection is objective because it is always based on choices. In this case, the choices have several levels.

There is also a third factor to consider, that cannot be circumvented, but which can, to some extent, contaminate the concepts achieved: the orientation and familiarity of the designers with the viewpoints of the advisors, namely the realistic perspective, with tendency to promote products of daily use, sometimes close to normal and banal, objects that serve to analyze and to portray our era, but also promoting design has a comprehensive tool to analyze the problems and interactions between material, environment, economy, culture and ethic [6].

The process used in the course was divided into three fundamental phases: in the first, investigation and concept, it was investigated the original object and its context, the current products with similar functionalities, and created the first proposals and concept sketches; in the second phase the product is developed, initially in general drawings, then more detailed, followed by volumetric models and scale models; in the third phase, and after fine-tuning the details, the three-dimensional CAD models, the final renders, the post-production of the images and posters of the final products were developed. In some cases, the respective packaging project was also designed.

\section{Design Approaches}

The student's approaches were fundamentally classified into three main types: 1 Evolutive design, maintaining the essential elements of the original, formally and functionally, trying to adapt to the current reality; 2 - Functional approach, maintaining functions, but with less fidelity to the original form; 3 - Formal approach, keeping references to the original form, but adapting the product to new functions, very different from the original pieces. 


\subsection{Evolutive}

Evolutive design can be characterized by a non-interventive approach, by a strong acceptance of existing products, by their valorization, occasionally promoting small changes that improve them without radically altering their character. The idea is related to existing products, with their improvement, creating the new one of existing references, appropriating the acquired knowledge. Hecht calls it appropriation [7] without giving it the sense of plagiarism, on the contrary, defending the clarity in the connection between the original product and the new product, respect and recourse to collective knowledge gathered over generations [4] than a copy.

According to Gustav Jung [8], this collective knowledge is inherited in the collective unconscious, the only component of the inherited psyche, common to all, where we store the most powerful memories and ideas, from religion to ethics, but also images which we all share and are familiar with. This idea of identification, of familiarity, belongs to each user, but also refers to knowledge accumulated over generations, rooted in their culture by repeated coexistence. Jasper Morrison defends the evolutionary step instead of the creative leap, the incremental improvement, projecting something that improves what already exists [9]. These principles are also based on the idea of not being invited to re-learn the interaction with the products, the simplification provided by communication and product honesty, allowing us to operate intuitively with the object [10]. The evolutive approach also invites the slowdown [11], by opposition to the speed and intensity of life today.

Among the current recognized products that fall into this category is the Déjà $\mathrm{Vu}$ stool, by Naoto Fukasawa, produced by Magis. In this stool - which incidentally was the origin of the exhibition and Super Normal book, according to Mateo Kries [12], one of the most important moments of recent design -, the form is recognized, but the material is new. The change is obvious, assumed, even excessive, in the loss of comfort transmitted by natural materials [13], but the form and function are kept intact. Socrates, from Jasper Morrison to Alessi, exemplifies evolutive design through functional improvement, celebrating the original corkscrew [14], reintroducing a typology that was disappearing [15]. This type of approach, even if looking to add something new, but not focusing on innovation, tends to be the most similar to the originals.

Thus, evolutive projects were considered as those in which the designer's option was to maintain a strong connection to the original, keeping the general forms of the original artefact, insisting in a similar or very close function, making slight changes or additions, functional improvements or soft formal modernizations, which allow a better integration of products to contemporaneity. Due to the need to use black clay, the only rule of work, the material change is one of the most repeated interventions, although in most cases not as present as in Déjà Vu. In some cases, as in the Pim Pam Pum (a) project, the material change is essentially reflected in color, as the original is also made of clay, but in orange. Through the new color, black, the author emphasizes the search for neutrality and invisibility, either from the perspective of Product as Landscape [7] or from a non-interventional perspective [16], which accentuates the communication of the project. In this project, the proposed use is like the original, but there are still other small evolutions, being added a piece to the set, proposing to serve and to cook more quantity and variety of food, always keeping the shape and function very close to the 
originals. In the Chopping Bowl (b) project, the reference to the original shape is perfectly visible, being, however, separated into two components, the black clay base and the wooden top. Besides the addition of a new material, a functional improvement is added, allowing the centerpiece to be raised to two different positions to ease cutting, by chopping. In both the OST (c) and Nigrum (d) designs, the function is kept very close to the original, with a second piece being added, together with the first one in wood and, not changing the original shape, increasing the possibilities of use, simplifying the profile, and thus seeking to fit into a contemporary table. In the FAI (e) project the addition of a new piece, once again in a new material, seeks to improve the use, avoiding contact with the conductive clay heated by the roasting. Multipurpose Bowl $(f)$ regularizes the geometry of the original, the Bow Set $(g)$ multiplies the plates and resizes them to provide a complete meal, Cousins $(h)$ recreates the three bowls in different materials, Ilusion (i) hides a structure, Biwine $(j)$ turns a spoon to drink in a bottle, and $J u g(k)$ tries to update a jar, all looking to adapt the new products to the new needs without a significant change on the original shape, keeping a clear and easy remission to the originals and the culture that they carry. In all cases the strategy seems to refer to "the evolutionary step" referred to by Morrison [4].

\subsection{Functional}

The second most used approach is functional orientation, seeking to maintain an equal or very similar function to the original use, paying less attention to formal resemblance to the original, most of which is recognizable only by volume, away from details, subtleties, and especially the more traditional character of the original object. The greater focus on function often leads to the devaluation of form and its elimination, resulting in the adoption of the simplest shape. This relation between function and reduction of form, this reference to a more basic geometry, is commonly associated with functionalism. One of the observations made to this approach, rooted in late modernism [17] is that it has become a language, that reduction has become an end and eliminated links to past. According to Tim Parsons [14], the separation between aesthetic and poetic cleansing abruptly and irreparably depletes and diminishes meaning. The existence of a basic form, of an original object that transports this past, somehow dilutes this formal effect, of reduction, of minimalism, although it is noticeable in many cases, a certain domain of the formal reduction on the object [18] and a desire to return to the primary use and elemental appearance of cyclically repeating objects [19]. Although this influence of a modernist approach is clear in the investigations presented during the process, this influence seems to arise more through present products of Nordic origin, simple geometries and natural materials, symmetrical but smooth, circulating in a space between the historical recovery by the postmodernism and modernist formal simplification [5]. 
The focus on function and consequent formal reduction, the greater liberation from the original form, seems to make the connection with the original objects less obvious than the evolutionary approach, resulting in more subtle relations, but giving rise to equally interesting products. On the other hand, with this greater break, highlights the connotation with an idea of modernization, contemporaneity and progress. In Coffea St (l), the search for a similar function, heating and serving a liquid, gives rise to a product with similar volumes, a more geometric appearance, but a much greater complexity, more components and technical implications in the production and use, seeming, on the other hand, more efficiently respond to current needs and better reflects the complexity of the world today. The greater liberation of the original from this type of approach makes the project more available for contamination by other influences such as coffee pots. In the Kaldi $(\mathrm{m})$ project, the transformation of the functional focus into formal simplification becomes even more evident, with the geometry to be used more purely, orthogonal and clearly minimal. In Simetea ( $n)$, a project that explores symmetry, simplification and mastery of the reduction language also become evident, with a clearly geometric approach, only softened by curved corners, making it look more contemporary. The same reduction game is available in Couple (o), Double Pan (p), Hydra (q), Todo (r) or Boni (s).

\subsection{Form}

In this third type of approach, inspiration is essentially formal. The designers seek to refer users to the original by copying a recognizable shape and adapting it to an object of different typology. This approach refers to the expression Without Thought by Fukasawa [20], who argues that the recognition of form reminds us about the original object, inviting unconscious action. He explores it on CD-Player, compact disc player with resemblance to an industrial fan, inviting the gesture of activation by pulling the wire. According to Masato Sasaki [20] this type of approach is linked to the idea of affordance, a term coined by James J. Gibson in the ecological psychology and which refers to the ability of objects to communicate potential, to invite or allow an action.

The Banzo $(t)$ project is precisely exploring the gesture of greeting by raising and turning the hat, repeating it in a cup of coffee. It becomes more interesting because it is one of the few projects in which the object selected has no material relation to the universe of the exercise, which makes it more challenging. The resulting form is that of the hat, the formal reference is obvious, but even the change of scale makes the difference more provocative. The eventual violence of the difference is mitigated by the gentle subtle formal treatment of Japanese inspiration, referring to the balance between past and future expressed in the products of Sori Yanagi. In other projects, exploration of the original shape is handled more directly. There is a clear mimic of the form and an adaptation to current needs, being intentionally ignored and avoided the relation with the original function. In Trawl $(u)$, the project in which perhaps this distance is smaller, the original butter dish is transformed into an exhibitor. There is a process of simplification and another of multiplication, creating three pieces with multiple possibilities of use, overlapping or placed on ladder, which highlights the difference with respect to the original function. The brutalist treatment seems to further accentuate the focus on formal inspiration, increasing the monolithic character of the piece, making it further away from the 
fragility of the original artifact. EspaBoard (v), a board inspired by "espadela", transforms the silhouette of an artifact that was used to brush and filter the wool in what it seems to be, in the light of today's life: a board for food. The formal appropriation is absolute, and any functional relation with the original is totally erased. The same is true for Cork Box $(w)$, a project in which a stool turns into a support table with the possibility of storing books or small objects, exploring the neutrality of the material to highlight the color of the objects that can be stored in it. The Lamp Cup $(x)$ also uses the original shapes, inverting the shells in order to build a lamp, apparently inspired by the PH series of Poul Henningsen or the latest Levels Lamp of the collective Form Us With Love. In JabBlock (y), the original shape is extruded, creating a totally different volumetric object, adapting it once again to a new utility, sticking knives. In Calabash (z), the function is also changed, but the shape maintains full connection to the original.

\subsection{Processual}

If 26 of the 28 products seem to fit directly into the three categories mentioned, two projects, not ignoring each of these approaches, maybe exploring little each one, focus on less typifiable and less visited approaches.

The project Bisalhas ( $\mathrm{aa}$ ) can initially be interpreted as a functional project, because it exploits a function of the original, that of container, apparently only adapted to present needs. On the other hand, it also keeps details that refer to the curves and handles of the original. It seems to seek to balance the two features in equal proportion, formal and functional, but what really dominates it, and which possibly makes it more interesting, is the way its center is freedom of use, a concept much more deeply linked to a social approach, much more focused on contemporaneity, on the way we live today, than on more practical approaches. The contamination of this contemporaneity and complexity is particularly clear in its influence suffered from Ovale, mirroring its rational regularity and desire for simplification, but also its smoothness, like time-smoothed pebbles [21], asymmetry and irregularity [22] or even imperfection, as in the Misfit sought by Hella Jongerius [23] or in Officina de Ronan and Erwan Bouroullec, which seek to replicate the defects caused by artisanal work. The relation with the original turns out to be more social rather than formal or functional. In String $(a b)$, the function is radically altered, so one could assume that the inspiration is formal. But the form is also subverted, being explored only one detail, which, although imperceptible in the original, makes the relationship subtle, as something that is discovered, that is not afraid of not being understood and does not need to scream. The wire, central in the new lamp, and the vertical space, create an unusual and interesting connection with the original, also avoiding previous cataloging's, which remind us how the reference can sometimes be only in detail and not in the whole, as in Milk NA1 from the Norm.Architects, referring us to the Nordic stool for material and number detail of legs. In these two projects, there is no direct formal appropriation, but only of details the authors considered central. There was no direct functional link, because even in the case where the function is similar, the proposed form of use, which is the center of the concept, is significantly different. The originals seemed to serve more as inspiration than as goal. 


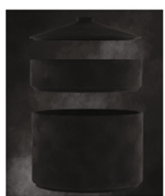

a) Pim Pam Pum

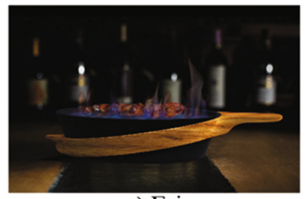

e) Fai

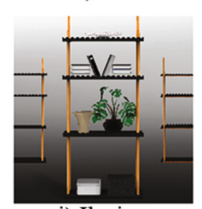

i) Ilusion

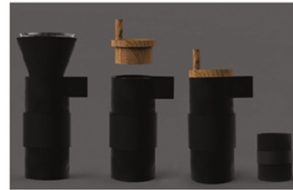

m) Kaldi

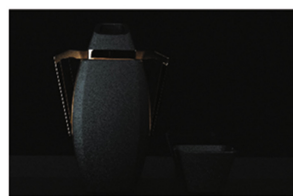

q) Hydra

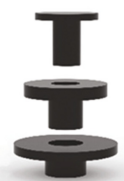

u) Trawl

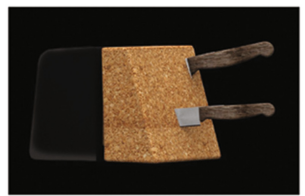

y) JabBlock

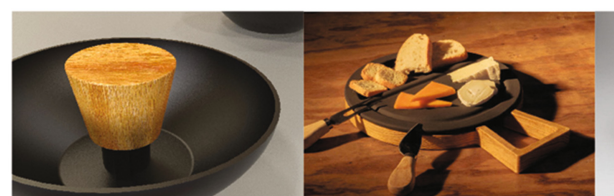

c) OST.

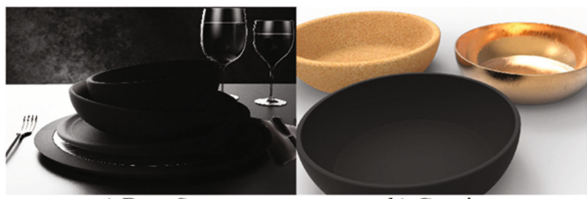

g) Bow Set

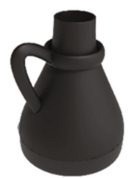

k) Jug

h) Cousins

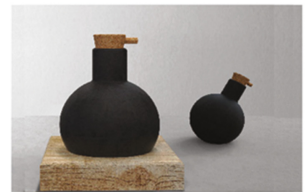

j) Biwine

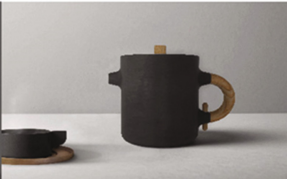

n) Simetea

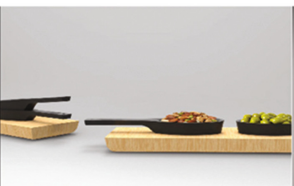

r) Todo

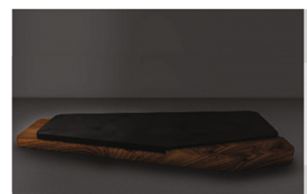

v) EspaBoard

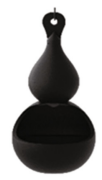

z) Calabsh o) Couple
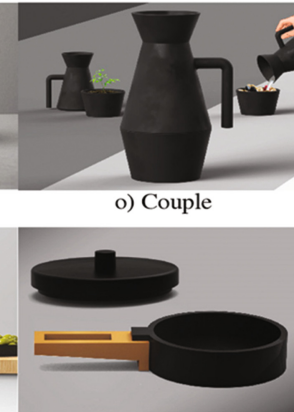

s) Boni

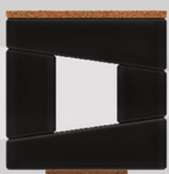

w) Cork Box

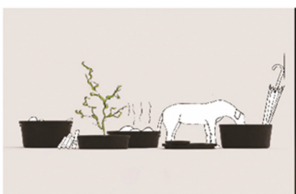

aa) Bisalhas

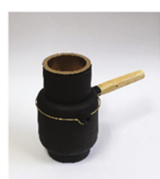

1) Coffea St d) Nigrum

Fig. 1. Projects, from top left to bottom right, by Nuno Viola, Karolina Wojtaszek, Jennifer Santos, Tiago Sieiro; Daniela Pereira, Milena Ziemkiewicz, Sofia Oliveira, João Camões; Rodolfo Alves, Daniel Couto, Catarina Caldeira, Miguel Batista; Natalina Carmo, Ana Vieira, David Pessanha, Emilia Placzek; Ângela Geraldes, Pedro Pereira, Cláudia Azevedo, Manuel Magalhães; Martyna Sodel, Rita Pereira, Miguel Rocha, Sofia Carreira; Luís Lopes, Hélia Pires, Daniel Catarino, Ana Salgueiro. 


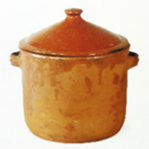

a) Cooking Pot

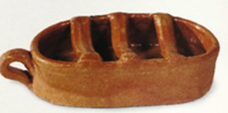

e) Chorizo Roaster

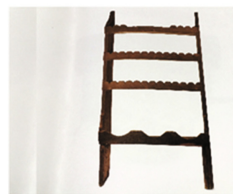

i) Kitchen Shelves

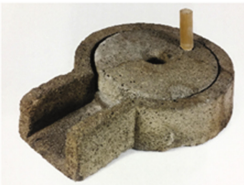

m) Coffee Mill

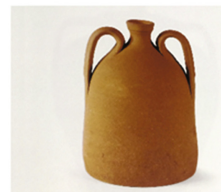

q) Water Pitcher

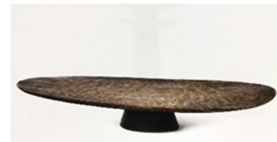

u) Butter Plater

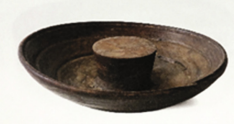

b) Chopping Bowl

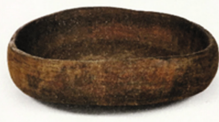

f) Wooden Bowl

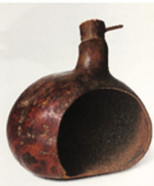

j) Wine Scoop

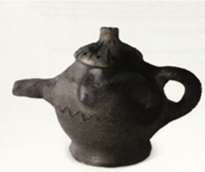

n) Teapot

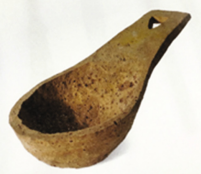

r) Drinking Scoop

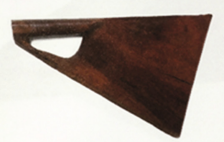

v) Espadela

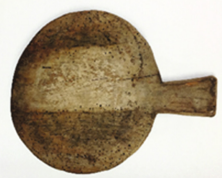

c) Francela

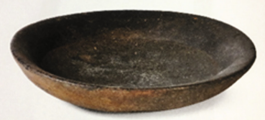

g) Wooden Bowl

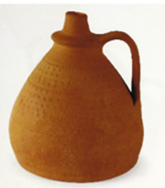

k) Water Pitcher

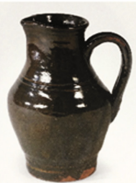

o) Jug

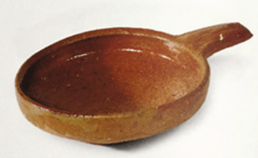

s) Frying Pan

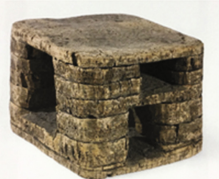

w) Cork Stool

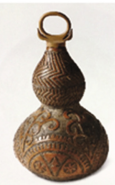

z) Water Flask

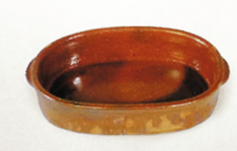

aa) Roasting Dish

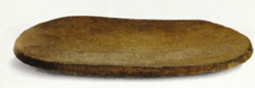

d) Butter Plater

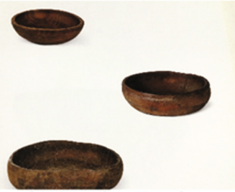

h) Wooden Bowls

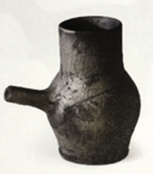

1) Coffee Pot

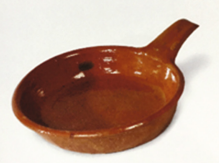

p) Frying Pan

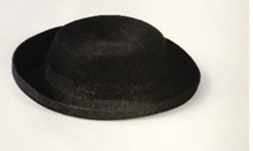

t) Hat

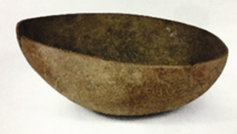

x) Wine Scoop

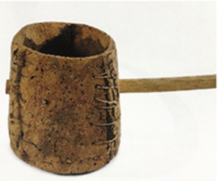

ab) Cork Water Scoop

Fig. 2. Objects from the National Museum of Ethnology selected by Jasper Morrison and that were used by the students as inspiration (Adapted from the book The Hard Life) 
The projects appear to be processual, not to be part of a very definite strategy and have been defined by the process itself. They are defined by the doubts that have prevailed over much of their development, in a non-linear process, with jumps, accidents, personal experiences [24] and chances [25]. These processes, which are more winding, have resulted in more original concepts, since they objectively do not fit into any typology so easily. On the other hand, it will be possible, unlike in the more defined categories, that remission to the originals may become less immediate.

In Fig. 1 is presented the projects, identified by name and letter, and, in Fig. 2, the objects selected by Morrison and by the students to serve as inspiration, also identified by letter in order to enable correspondence to the projects.

\section{Conclusion}

It is not suggested that any approach is seen as right or wrong. The same way the project developed was more oriented on the process and quality of the solutions, this article focuses more on the analysis of the paths chosen, in the search to understand how a group of young designers interprets and projects from objects that, not being part of their day to day, still belong to their individual memory, as also to collective and national culture. They refer to a general idea of the essentially rural past, but also to people and places that are different for each student. The challenge implies many dimensions, but the one that seems to have been central, and that allowed the cataloging of the works, is the approach, the strategy chosen to redesign each object. The choice of an evolutive, functional or formal path turns out to be the fundamental decision that, as demonstrated in the paper, turns out to be decisive in the definition of the language used in each project. In the evolutive approach results in versions closer to the originals, in the functional tends to a dominant simplification. The language, the formal lexicon of each project, rather than a response, tends to be coherent with the conceptual decision, with the choice of one of the three paths listed here.

While it is true that the cataloged approaches are clearly different, this does not imply that the projects have been developed with full fidelity to only one of these principles. In some projects, the option places them only in one of the approaches, others move in gray territory between two, or even three, perspectives. It can be concluded that none of the approaches statistically dominated the student's choices: eleven projects were considered evolutive, eight of functional focus, seven of formal inspiration, with two of the projects placed outside this cataloging. This seems to reflect diversity and the absence of a dominant thought or aesthetics, confirming a desire for free thought.

Acknowledgments. To Bisarro, to the National Museum of Ethnology and to the Students of the Master Degree in Industrial Design at University of Beira Interior. 


\section{References}

1. Morrison, J.: The Hard life. Lars Muller Publishers, Zurich (2017)

2. Silva, J.R., Sequeira, J.L.: Ficha de Inventário de Património Imaterial $n^{\circ}$ INPCI SU 2015 001. DGCP, Lisboa (2015)

3. Hara, K.: Designing Design. Lars Muller Publishers, Baden (2007)

4. Fukasawa, N., Morrison, J.: Super Normal. Lars Muller Publishers, Baden (2007)

5. Sudjic, D.: The Language of Things. Penguin, London (2009)

6. Vicente, J., Frazão, R., da Silva, F.M.: Sustainable product design and the wood furniture sector. In: Rebelo, F., Soares, M. (eds.) Advances in Ergonomics in Design. Advances in Intelligent Systems and Computing, vol. 588, pp. 762-772. Springer, Cham (2018)

7. Fukasawa, N., Hecht, S., Colin, K.: Industrial Facility: Product as Landscape. Industrial Facility, London (2005)

8. Jung, C.G.: Synchronicity: An Acausal Connecting Principle. Routledge and Kegan Paul, Oxford (1972)

9. Morrison, J.: Il Progetto Necessario. Domus, 977 (2014)

10. Terstiege, G.: Making things visible. Vitra Mag. (2009)

11. Borges, A.: Normal, memória, invisibilidade e significado nos objectos. Tese de Doutoramento. Universidade de Aveiro, Aveiro (2015)

12. Dornbracht Conversations. https://www.dornbracht.com/pt-pt/company/press/news/2011/ ish/culture-projects/01_dornbracht_conversations_3/

13. Baudrillard, J.: Le Système Des Objets. Verso, London (2005)

14. Parsons, T.: Thinking: Objects: Contemporary Approaches to Product Design. AVA Publishing, Lausanne (2009)

15. Morrison, J.: Everything But the Walls. Lars Muller Publishers, Baden (2006)

16. Bil'ak, P.: Underdesign, Overdesign, Redesign. Dot dot dot (2001). http://www.icograda. org/feature/current/articles36.htm

17. Thackara, J.: Design After Modernism. Thames \& Hudson, London (1988)

18. Schwartz-Clauss, M., von Vegesack, A.: Die Essenz der Dinge. Vitra Design Stiftung, Weil am Rhein (2010)

19. Pizzi, M., Mendini, A.: Piccola storia del Divano. Abitare 493 (2009)

20. Fukasawa, N.: Naoto Fukasawa. Phaidon Press, London (2007)

21. Koivu, A., Bouroullec, R., Bouroullec, E.: Ronan and Erwan Bouroullec: Works. Phaidon Press, London (2012)

22. Rawsthorn, A.: Japanese Design Group Nendo Hones Its Unusual Charm. The New York Times, 29 January 2012 (2012)

23. Schouwenberg, L., Antonelli, P., Rawsthorn, A., Boom, I.: Hella Jongerius: Misfit. Phaidon Press, London (2010)

24. Kries, M., Lipsky, J.: Konstantin Grcic: Panorama. Vitra Design Museum, Weil am Rhein (2014)

25. Scholze, J., Ross, J.A:. No cosy corners. Disegno 4 (2013) 